\title{
WFH and LFH Phenomenon: Redefined Active and Passive Space in Residential
}

\author{
Popi Puspitasari ${ }^{1}$, Resa Hadi Nugraha Sodikin ${ }^{2}$ \\ Jurusan Arsitektur, FTSP, Universitas Trisakti ${ }^{1}$ \\ Jurusan Arsitektur, FTSP, Universitas Trisakti ${ }^{2}$ \\ \{popi@trisakti.ac.id ${ }^{1}$, resasodikin@gmail.com²
}

\begin{abstract}
The residence's rooms have their respective functions according to the needs and preferences of family members. The rooms function effectiveness indicated by the availability of facilities in the form of furniture to support activities arranged in such a way as to match the priority range. However, there are individual rooms where the frequency of use is low (passive), and some are high (active) so that the spaces are no longer efficient evenly and optimally utilized. Work From Home (WFH) and Learning From House (LFH) due to the Covid-19 pandemic forces every family member to have activities at home throughout the day, thus providing an opportunity to recognize which spaces were active and passive. The research aimed to indicate the active and passive spaces in the house during WFH. The qualitative method with behavior mapping was considered suitable to meet this need. The research population was families, with a total sample of 30 architecture student families, FTSP, Trisakti University. As an informant, each student described the pattern of daily activities for his/her family member within one week during WFH, which was complemented by explaining the types and motives of the activities. The results showed that; 1) passive and active spaces related to the types of primary, secondary, and tertiary needs; 2) the movement patterns of inactive spaces indicated the nature of feminine and masculine activities; 3 ) the changing passive space into active one related to the transfer of formal activities to earn a living into the house. The transfer of work and study activities from outside into the house during WFH and LFH has reduced and increased activity in the house's specific rooms, integrating the real world and the virtual world. This reduction and increase related to the Covid-19 protocol, privacy, preferences, and priorities.
\end{abstract}

Keywords: WFH; Active and Passive space; Residential

\section{Introduction}

The effectiveness of rooms' function is determined by the frequency and capacity of use to accommodate both goods and people. The relatively high frequency and capacity of a room for user activities encourage the perception that the room is active; otherwise, a passive room is a low frequency of use. A room is declared effective and efficient if each room's area 
accommodates a minimum of primary activities in daily needs. Investigating whether a room is active or passive, or in which corner of the room in an active or passive house, will be detected if all house residents are active all day in the house. Work from home (WFH) and Learning From Home (LFH) during the Covid-19 pandemic provided an opportunity to carry out such investigations, in which all family members were forced to do activities indoors all day long.

Behavior mapping is widely used in various fields of science in the micro to macro areas. Mental conditions stimulate behavior mapping. In architectural spatial studies, cognitive mapping is fundamental to construct a map of people's movement (as observed objects) in a particular spatial setting based on people's attributes and relative locations to objects in their environment. The nature of people's movement in specific settings is determined by the person's considerations about safety, speed, preference, to take a particular route according to destination or purpose. As a process, behavior mapping is not constant or changes according to age changes or the development of experience/learning. The substance of behavior mapping can be related to the distance and development of spatial cognition and spatial preferences and representations, and geographical orientation in spatial relation [7]. Spatially, a person's movement is related to the individual's subjective considerations (preferences, meanings, the perceived value of space, and binding procedures). Preference for a particular space is influenced significantly by gender, age, environmental situation. Other aspects are considerations of privacy, opportunities for interaction, autonomy, the physical comfort of the environment (thermal, physical, or visual)[8]. Meanwhile, spatial value's meaning and perception are often the manifestations of belief rituals and economic profit orientation [9].

The development of lifestyle and technology has changed human habits in carrying out daily life and work. Today, many companies offer to facilitate household activities. A coworking space facility is an option to work outside the home that allows people to work in an inspiring work environment [10]. Accessibility and atmosphere/interior are the most important characteristics when choosing a particular co- working space [11]. The people express their motivation, inspiration, and productivity in the real world and the virtual world. Big-data availability in the digital world allows consumers to live their day - communicating, exploring, buying, sharing, searching - to create their vast data footprint that creates their own virtual spatial space [10]. This context has cut the physical spatial distance so that individuals can control the movement of their daily life in both real and virtual worlds. The Covid-19 pandemic phenomenon has forced the emergence of WFH and LFH policies, which impact decreasing physical and psychological endurance, changing daily activities' structure, and enhancing cyberspace life. Exercising, walking, gardening, doing hobbies, and caring for children are the WFH's activities considered most significant to increase affective benefit [12].

This study aimed to determine active and passive spaces by mapping the behavior of family members in everyday life during WFH and LFH. Several previous studies found that 1) the productivity of working at home during Covid has decreased the productivity of working in the office; 2) it was identified that several types of office work unavailable at home; 3) during WFH, workers balanced routine activities of daily life with office work: 4) focus of office work was distracted by homework [1]. The Covid 19 pandemic impacts changes in work practices, interaction and social psychology, demographic characteristics, individual differences, and organizational norms [2]. Social behavior to reduce the risk of infection related to education level. Highly educated respondents $(45 \%)$ who comply with work from home (WFH) were higher in number than respondents with low education (11-17\%). However, these differences were not related to health behavior differences but related to the inability to practice social distancing in the workplace [3]. The Covid 19 pandemic also had an impact on 1) the value and 
status of work, which affected changes in the supply and demand for jobs; 2) work-from-home effectiveness in terms of job and individual characteristics; 3 ) increased segmentation of the labor market, which allocates workers to "good jobs" and "bad jobs" and the contribution of job segmentation to inequality [4]. In the health sector, the pandemic impacts the increasing anticipatory attitudes of family members in the home, whose behavior pays more attention to the Covid-19 protocol [5]. Staying at home due to a pandemic causes health anxiety, financial worries, and the need for social support [6].

In this study, WFH and LFH as the impact of the Covid-19 pandemic were used as an essential momentum to observe the behavior patterns of family members in carrying out their daily life optimally at home. These daily activities occur in rooms in the house; if it is not WFH and LFH, the observed behavior patterns may not be optimal. The research's hypothetical statement is that WFH and LFH cause a reduction and increase in activity in specific spaces in the house.

\section{Methods}

The research objective was to identify the functional space and passive space in the house by mapping the occupants' behavior during WFH and LFH. The research approach that considered appropriate was inductive-qualitative, which combined images of behavior mapping accompanied by explanations of the person's motives when carried out activities. The studied population was households with a sample of households living in medium and large houses completed by a living room, family room, dining room, bedroom, kitchen, toilet, yard, or indoor/outdoor garden. Informants were 30 architecture students, FTSP, Trisakti University. The ability to understand basic research knowledge understood by students guaranteed that $85 \%$ of the data validity was assumed to be reliable. $15 \%$ belief in the invalidity of data came from incomplete verbal data.

Data were in the form of maps of the daily activities of family members made by each informant and accompanied by an explanation of the activity motive. The data consists of activities of family members, places of activity, time of activities and behavior patterns. Information was obtained based on the observation of each informant's during seven days in the morning, afternoon, and evening. Graphical presentation was used to accumulate each activity's frequency and duration. The analysis scope included analysis of movement in space, analysis of space categorized as active and passive, and analysis of the meaning of verbal explanation. The three analyzes were then interpreted in an integrated manner. As a qualitative inductive research, analysis was carried out by classifying units of information into certain themes, then logically linking these themes into concept formulations. 


\section{Results}

The results showed three themes found through the research: 1) passive and active spaces related to the types of primary, secondary, and tertiary needs; 2) the movement patterns of inactive spaces indicated the nature of feminine and masculine activities; 3) the changing passive space into active one related to the transfer of formal activities to earn a living into the house. Each explanation of the research results is as follows:

1) Passive and active spaces related to the types of primary, secondary, and tertiary needs

The residence studied consists of a living room, family room, dining room, bedroom, study room that was united with the bedroom, kitchen, garage, yard, garden, ironing room, toilet and laundry room, warehouse. These spaces can be classified into primary, secondary, and tertiary based on the frequency of use and level of need in routine daily activities. Rooms with primary categories were rooms that accommodate routine needs or obligatory activities that cannot be replaced by others, for example, rooms that accommodate sleeping (bedroom), bathing (toilet), cooking (kitchen), and eating (dining room).

Rooms categorized as secondary are alternative rooms that can be replaced or unified with other rooms, including family gathering rooms, workrooms, and study rooms. During WFH and LFH, the bedroom, living room, dining room, or family room used for work/study rooms. Tertiary rooms are complementary rooms that support the primary or secondary rooms include warehouse, garden, prayer room, guest room, garage. The living room was passive before WFH. In everyday life, residents mostly take advantage of the living room to crossway from the family room to the yard. However, male parents made this seldom-used space an option for WFH.

The duration of use indicates the active and passive spaces. Figure 1 shows the difference in the duration of space usage. Before WFH, for 8 hours, the father worked outside, and the children at school and the house's rooms relatively only accommodated routine household activities and worship. During WFH, the 8 hours duration is diverted into the house so that several rooms in the house become more active due to the added function of WFH and LFH besides the need for relaxation.

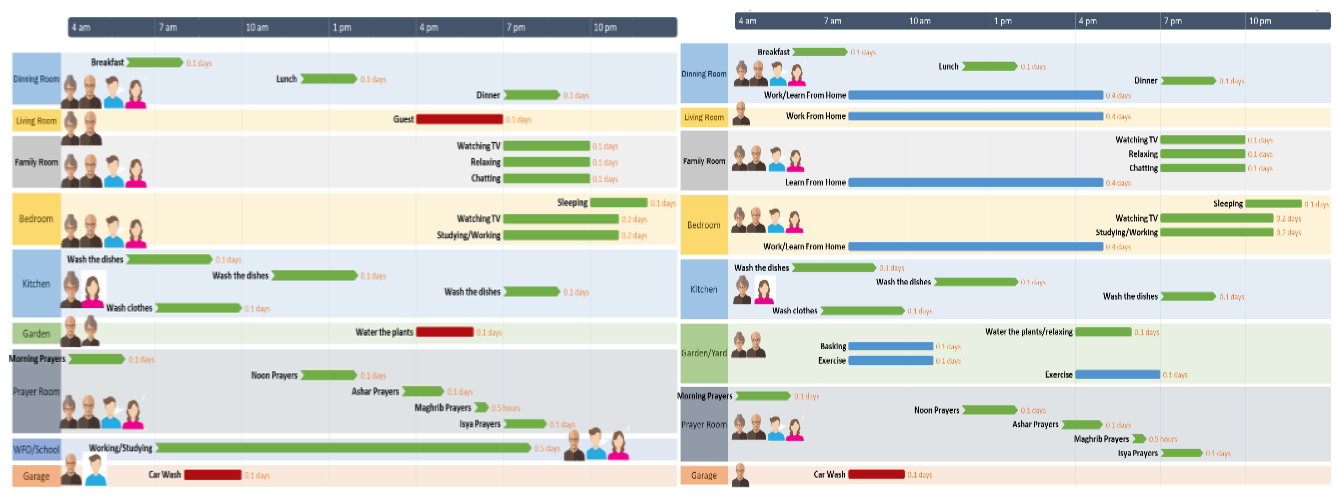

Figure 1. Duration of rooms use when WFO (left) and WFH (right)

1) The movement patterns of inactive spaces indicated the nature of feminine and masculine activities

Active rooms characterize masculinity and femininity. The feminine space is the path between the bedroom, living room, dining room, kitchen, and laundry area. Meanwhile, 
masculine space is the path between the bedroom, dining room/family room, garage, and garden, including the warehouse. However, there are exceptions to an incomplete family. For example, the occupants consist of only boys and grandmothers or single parents with one daughter; the boys carry out activities that are generally carried out by women so that the rooms can no longer be distinguished between feminine or masculine. A family with a father who worked outside the home (self-employed) during the Covid-19 Pandemic. The time for doing activities at home for the father is dominant after 4:00 p.m. with more time in the bedroom, living room, dining room, and indoor mini garden. Mothers with housewives' status and who have maids spend more of their daily time in the bedroom, dining room, prayer room, and mini indoor garden. Unlike the ancient houses that did not use household helpers, the kitchen and bathroom were very functional rooms for homemakers. Children with college student status are active in the bedroom at hours, according to online lecture hours. Likewise, mothers who have online businesses use almost all rooms.

The changing passive space into active one related to the transfer of formal activities to earn a living into the house

Changes in passive rooms to be active or very active during WFH are related to the number of families, preferences, privacy, house size, room variations. Unlike in a narrow house, large houses with complete room types allow family members to choose the rooms according to preferences and privacy considerations. This condition encourages family members to carry out WFH (Work From Home) and LFH (Learning From Home) activities in the family room, dining room, and living room. The living room is a space that decreases and also increases activity during WFH. Reducing the living room's activeness is related to reducing families receiving outside guests in anticipation of physical distancing. However, the living room's liveliness increases when one family member uses the living room as a WFH room, especially for fathers. Mothers use all rooms for online business activities, but the most widely used rooms are the family room and bedroom. While children with college student status use the bedroom actively for learning. For those who do not have a desk, the bed is a proper place to study where study and rest are carried out alternately. The increase in active activities in the room during WFH is directly proportional to working hours in the office and class schedules.

\section{Discussion}

Active and passive spaces during WFH are related to 3 (three) things, namely activities, time, and space, each related to one another. The family's economic status determines the dimensions, variety, and ownership of supporting space (for activities that are considered secondary or tertiary). Family members carry out daily activities based on priority considerations determined by the ease of reach of furniture or items that support smooth activities and the level of need. There are indications of the grouping of primary, secondary, and tertiary activities. All three are related to the time, which appears in the frequency of repetitive activities (household, livelihoods, relaxation, and worship routine activities) and duration.

Based on the patterns and types of activities, there is a grouping of activity routes that are feminine (household activities), masculine (livelihood activities), and neutral (relaxation and worship activities). The age of family members (children, youth, and parents) determines the types of livelihood activities, whether WFH or WFO, and children's learning activities (LFH). Individual spaces by adult actors (students) or parents (workers) are related to privacy needs 
or preferences. The availability of private space depends on the dimensions and variety of space; for example, a small house may not provide individual study or workspace, so as a consequence, the individual uses other multifunctional spaces. During WFH, family members do relaxation and stress relief activities in the indoor mini-park or outdoor garden due to anticipating health and getting out of boredom. Under Covid 19 protocol consideration, the family avoids accepting people outside the family, resulting in a reduction in the living room frequency.

Through previous research, "The Concept of Space Inhabitation:" Nearly Adequate, " researchers tried to explore the function of space in a simple fabricated house [13]. The research findings showed that a simple house with limited space and dimensions, while various daily activities were complex, caused residents hardly to regulate their activities' privacy. As a result, the whole space became multifunctional, and what family members did was regulate the use of space-based on-non-verbal agreements. Family members' survival depended on their flexible attitude through the principle of "just enough"/"nearly adequate" so that individual privacy needs and preferences were challenging to define.

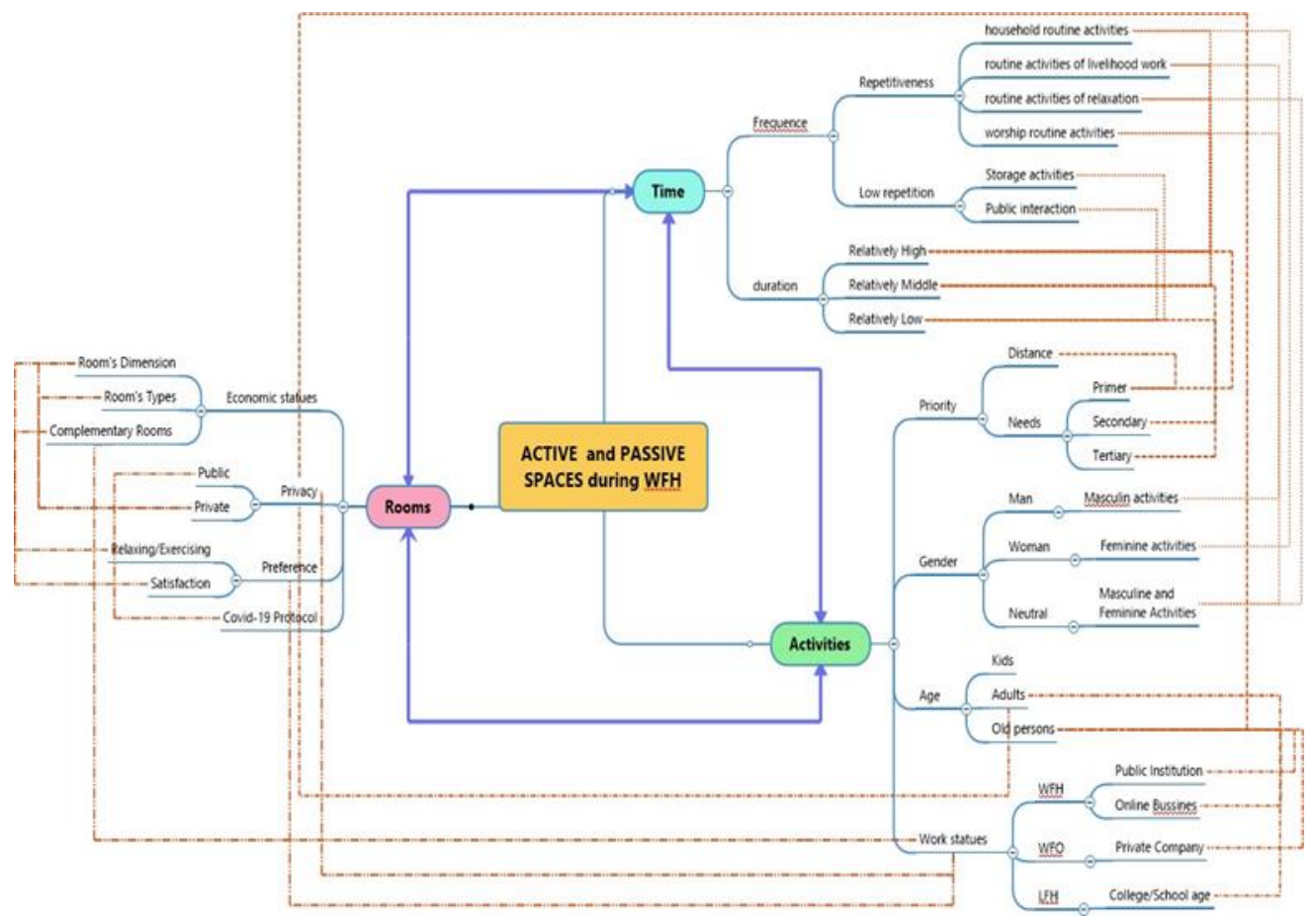

Figure 2. Conceptual formulation of the factors that influence the emergence of active and passive spaces in the house during WFH and LFH

Referring to the current conditions, the development of digital technology used during WFH has made it easier to control any activity through electronic networks so that private and shared spaces in the house facilitate the integration of the real world and the virtual world. Tull [6], Lades [10], and Power [12] found that psychologically WFH affects increasing stress and anxiety of family members and women's workload. Research published in this article found that stress relief manifested in increased relaxation activities in indoor mini-spaces and 
outdoor gardens at home. 


\section{Conclusion}

Activity, time, and spatial characteristics determine active and passive rooms in the house, the three aspects of which influence one another. Complex activities require various types of room, and each activity has a duration and time repetition. The limited space does not entirely reduce the complexity of the activities' types, but it is determined by the family members' flexibility and the agreement between them. The transfer of work and study activities from outside $t$ into the house during WFH has reduced and increased activity in the house's specific rooms, integrating the real world and the virtual world. This reduction and increase related to the Covid-19 protocol, privacy, preferences, and priorities.

\section{References}

[1] D. Mustajab, A. Bauw, A. Rasyid, A. Irawan, M. A. Akbar, and M. A. Hamid, "Working From Home Phenomenon As an Effort to Prevent COVID-19 Attacks and Its Impacts on Work Productivity," TIJAB (The Int. J. Appl. Business), 2020, doi: 10.20473/tijab.v4.i1.2020.13-21.

[2] K. M. Kniffin et al., "COVID-19 and the Workplace: Implications, Issues, and Insights for Future Research and Action," Am. Psychol., 2020, doi: 10.1037/amp0000716.

[3] K. Hoenig and S. E. Wenz, "Education, health behavior, and working conditions during the pandemic: evidence from a German sample," Eur. Soc., 2020, doi: 10.1080/14616696.2020.1824004.

[4] A. Kramer and K. Z. Kramer, "The potential impact of the Covid-19 pandemic on occupational status, work from home, and occupational mobility," Journal of Vocational Behavior. 2020, doi: 10.1016/j.jvb.2020.103442.

[5] A. Haleem, M. Javaid, and R. Vaishya, "Effects of COVID-19 pandemic in daily life," Curr. Med. Res.

[6] Pract., 2020, doi: 10.1016/j.cmrp.2020.03.011.

[7] M. T. Tull, K. A. Edmonds, K. M. Scamaldo, J. R. Richmond, J. P. Rose, and K. L. Gratz, "Psychological Outcomes Associated with Stay-at-Home Orders and the Perceived Impact of COVID-19 on Daily Life," Psychiatry Res., 2020, doi: 10.1016/j.psychres.2020.113098.

[8] R. M. Downs, D. Stea, and K. E. Boulding, Image and environment: Cognitive mapping and spatial behavior. 2017.

[9] R. Beckers, T. van der Voordt, and G. Dewulf, "Learning space preferences of higher education students,"

[10] Build. Environ., 2016, doi: 10.1016/j.buildenv.2016.05.013.

[11] P. Puspitasari, S. A. Djunaedi, and H. S. A. Putra, "Ritual and Space Structure: Pilgrimage and Space Use in Historical Urban Kampung Context of Luar Batang (Jakarta, Indonesia)," Procedia - Soc. Behav. Sci., vol. 36, pp. 350-360, Apr. 2012, doi: 10.1016/j.sbspro.2012.03.039.

[12] M. Weijs-Perrée, J. van de Koevering, R. Appel-Meulenbroek, and T. Arentze, "Analysing user preferences for co-working space characteristics," Build. Res. Inf., 2019, doi: 10.1080/09613218.2018.1463750.

[13] L. K. Lades, K. Laffan, M. Daly, and L. Delaney, "Daily emotional well-being 
during the COVID-19 pandemic," Br. J. Health Psychol., 2020, doi: 10.1111/bjhp. 12450 .

[14] K. Power, "The COVID-19 pandemic has increased the care burden of women and families,” Sustain. Sci. Pract. Policy, 2020, doi: 10.1080/15487733.2020.1776561.

[15] Olivia Yuni Rahayu; Popi Puspitasari; Indartoyo Indartoyo, "The Concept of Space Inhabitation: 'Nearly Adequate,"' LivaS - Int. J. Livable Space, vol. 1, pp. 29-38, 2016. 